\title{
Timing is everything for Nogo-specific antibodies
}

Current treatments for disability following a stroke focus on physical rehabilitation, as there are no effective pharmacological interventions that promote nerve regrowth. Reporting in Science, Schwab and colleagues now show that treatment with an antibody directed against Nogo-A (also known as reticulon $4 \mathrm{~A}$ ), if given before the start of physical therapy, can result in nearly complete recovery of forelimb function in a rat model of stroke.

Nogo-A inhibits neuronal regrowth. Antibodies targeting this molecule have been investigated in clinical trials as potential treatments for stroke, acute spinal cord injury and amyotrophic lateral sclerosis. Although only Phase I tolerability and dosing tests have been completed so far, animal experiments suggest that both nerve regrowth and plasticity, which can be encouraged by physical therapy, must be stimulated to allow functional recovery. Therapies that promote these two different processes might therefore need to be used sequentially, as nerve endings must be in close proximity before synapses can form between them.

Using a mouse model in which stroke was induced by microthrombi, the investigators tested whether the timing of antibody therapy and physical therapy influenced recovery. Animals had subtotal destruction of one sensory motor cortex, resulting in severe and permanent impairment of paw and limb function, particularly fine motor control. They were then treated with intrathecal injection of Nogo-A-specific antibodies or control antibodies, either before or at the same time as physical therapies that promote recovery of fine motor skills. Animals treated sequentially with Nogo-A-specific antibodies and rehabilitation recovered $80-100 \%$ of their pre-stroke forelimb function, whereas animals treated with control antibodies, or those that received both therapies simultaneously, recovered less than $40 \%$.

Neuronal regeneration is thought to occur from the growth of contralesional corticospinal tract fibres that cross the midline to re-innervate the stroke-affected side. Rats that were treated with the two therapies sequentially had more midlinecrossing fibres than did the other groups, thus providing histological support for the model of improved function. By contrast, mice treated simultaneously with both therapies had histological evidence of hyperinnervation, with overshooting fibre growth and aberrant termination patterns.

The investigators then used reversible nerve inactivation to specifically test where the regenerating neurons were coming from. Animals from the group that received the antibody followed by physical therapy were treated with two viruses: a virus encoding a neurotoxin downstream of the tetracycline promoter was injected into the stroke-denervated side of the cervical spinal cord, and a virus encoding the reverse tetracycline transactivator was injected into the contralesional premotor and motor cortex. Only neurons originating on the contralesional side of the midline with axons projecting to the denervated side would receive both viruses, and would therefore express the neurotoxin upon exposure to tetracycline. Indeed, animals injected with both of these viruses lost forelimb function when given tetracycline, and regained function upon tetracycline withdrawal. A similar system using different viruses and genes - but still with the injection of one virus on the strokedenervated side and one on the intact contralesional side - confirmed these results.

These data suggest that the sequential timing of the anti-Nogo-A therapy in relation to physical therapy is of critical importance and will need to be optimized in future clinical trials.

Megan Cully

ORIGINAL RESEARCH PAPER Wahl, A. S. et al. Asynchronous therapy restores motor control by rewiring of the rat corticospinal tract after stroke. Science 344, 1250-1255 (2014) 